\title{
RESISTENCIA DE SEMILLAS DEL HÍBRIDO DE MAÍZ HS-2 A COMPRESIÓN AXIAL
}

\author{
RESISTANCE OF HS-2 HYBRID MAIZE SEEDS TO AXIAL COMPRESSION
}

\author{
Carlos A. Villaseñor-Perea', Araceli Ramírez-Jaspeado ${ }^{2}$, Arturo \\ Mancera-Rico ${ }^{3}$ y Ma. del Rosario Venegas-Ordoñez ${ }^{1}$
}

\begin{abstract}
'Departamento de Ingeniería Mecánica Agrícola, Universidad Autónoma Chapingo. Km 38.5 Carr. México-Texcoco. 56230, Chapingo, Estado de México. ${ }^{2}$ COLPOSEED y ${ }^{3}$ Producción de Semillas, Colegio de Postgraduados. Km 36.5 Carr. México-Texcoco. 56230, Montecillo, Estado de México.
\end{abstract}

Autor para correspondencia (aracelirj@colpos.mx)

\section{RESUMEN}

La resistencia a compresión axial influye en distintas etapas del proceso de producción de semillas, lo que incluye la selección de progenitores, acondicionamiento y almacenaje, además del diseño de maquinaria y de las operaciones. En este estudio, realizado en 2013 en el laboratorio de materiales de la Universidad Autónoma Chapingo, Estado de México, se determinó la fuerza y deformación en los puntos de límite proporcional (LL), biocedencia (Y) y ruptura (R) de la semilla del híbrido de maíz (Zea mays L.) HS-2 de los estratos apical, medio y basal de la mazorca con humedades de 10 y $23 \%$ sometidas a compresión axial. Para ello, las semillas se comprimieron en una máquina universal de ensaye entre superficies planas y pulidas. Las semillas del estrato medio estudiadas a $10 \%$ de humedad, fueron más resistentes a la carga axial $(\mathrm{LL}=301 \mathrm{~N}, \mathrm{Y}=434 \mathrm{~N}$ y $\mathrm{R}=344 \mathrm{~N})$ que las correspondientes a los estratos apical y basal (LL = 258 y $238 \mathrm{~N}, \mathrm{Y}=345$ y $341 \mathrm{~N}$, y $R=248$ y $246 \mathrm{~N}$, respectivamente). Semillas con $23 \%$ de humedad presentaron mayor resistencia a la compresión que semillas con humedad de $10 \%$ ( $L L=429$ vs. $301 \mathrm{~N}, \mathrm{Y}=598$ vs. $434 \mathrm{~N}$, y $\mathrm{R}=568$ vs. $344 \mathrm{~N}$, respectivamente), además de una mayor elasticidad $(L L=0.827$ vs. $0.215 \mathrm{~mm}$ ) y desplazamiento en general ( $Y=1.165$ vs. $0.303 \mathrm{~mm}, y \mathrm{R}=1.215$ vs. $0.341 \mathrm{~mm}$, respectivamente).

Palabras clave: Humedad, estrato, fuerza, deformación, Zea mays.

\section{SUMMARY}

Axial compression resistance influences seed production stages, such as parent selection, seed conditioning and storage, and design of machinery and operations. In this study, conducted in 2013 at the Materials Laboratory of Universidad Autónoma Chapingo, State of Mexico, the strength and deformation at points of proportional limit $(\mathrm{LL})$, biocedence $(\mathrm{Y})$ and rupture (R) were determined in seeds of HS-2 hybrid maize (Zea mays L.). Seeds were subjected to axial compression at the apical, middle and basal ear strata at 10 and $23 \%$ moisture content. Seeds were compressed between two flat and polished plates by using a universal testing machine. Seeds from the middle stratum at $10 \%$ moisture content were more resistant to axial load (LL $=301 \mathrm{~N}$, $\mathrm{Y}=434 \mathrm{~N}$ and $\mathrm{R}=344 \mathrm{~N})$ than those from the apical and basal strata $(\mathrm{LL}=258$ and $238 \mathrm{~N}, \mathrm{Y}=345$ and $341 \mathrm{~N}$, and $\mathrm{R}=248$ and $246 \mathrm{~N}$, respectively). Seeds at $23 \%$ moisture content had higher compression resistance than seeds at $10 \%$ moisture content ( $L L=429$ vs. $301 \mathrm{~N}, \mathrm{Y}=598$ vs. $434 \mathrm{~N}$, and $\mathrm{R}=568$ vs. 344 $\mathrm{N}$, respectively), as well as higher elasticity ( $\mathrm{LL}=0.827 \mathrm{vs} .0 .215 \mathrm{~mm}$ ) and displacement in general $(Y=1.165$ vs. $0.303 \mathrm{~mm}$, and $\mathrm{R}=1.215$ vs. 0.341 $\mathrm{mm}$, respectively).

Index words: Moisture, strata, strength, deformation, Zea mays.

\section{INTRODUCCIÓN}

La resistencia a daño mecánico es un factor que condiciona distintas etapas del proceso de producción de semilla, las cuales pueden incluir desde la siembra de los progenitores, cosecha, secado, limpieza o beneficio y almacenaje (Basu, 1995; Desai et al., 1997), además de la siembra de la semilla por los agricultores. Usualmente se adecua el régimen de trabajo de los equipos de tal forma que la semilla sufra el menor daño posible. Por otro lado, el diseño de máquinas con frecuencia considera las propiedades físicas y mecánicas de los productos que ha de procesar (Mohsenin, 1986) con el propósito de realizar el trabajo de manera eficiente en el sentido de consumo de energía por unidad procesada y eficaz, para efectuar el trabajo de la mejor manera, sin dejar de atender la calidad de los productos.

Desde hace varias décadas, la producción de semilla mejorada tiene la finalidad de satisfacer las necesidades y exigencias de la industria que ha de procesar los distintos granos para la obtención de productos finales como pastas, harinas, hojuelas, alimento para ganado, aceites, etc. (Poehlman y Sleper, 2003), donde los estudios de resistencia mecánica pueden ser importantes. García-Lara et al. (2004) encontraron una correlación alta entre la dureza de la semilla y la resistencia al ataque por gorgojo (Curculionidae), aspecto que puede ser de relevancia en la sanidad y pérdidas durante el almacenaje de la semilla.

Los resultados obtenidos por algunos autores sugieren que la resistencia mecánica de las semillas está influenciada por varios factores, entre los que se puede mencionar la humedad, la madurez, el tamaño y la forma. Mesquita y Hanna (1993) encontraron menor resistencia a menor contenido de humedad en semilla de soya (Glicyne max), mientras que Narayan y Bilanski (1966) encontraron que la energía necesaria para ocasionar fractura en semillas 
de trigo (Triticum spp.) se incrementó de manera proporcional al contenido de humedad, de manera aproximadamente lineal; en este sentido, Multon (1981) y Mohsenin (1986) mencionan que un incremento en agua "ligada" a la matriz de proteína, almidón y pentosas, ocasiona que las semillas sean menos resistentes a la deformación y más resistentes al quebrado. En contraste, King y Riddolls (1960) encontraron que conforme hubo un incremento en el contenido de humedad durante la trilla, hubo un decremento en el daño visible pero también en el porcentaje de germinación, lo que indica que el daño fisiológico fue superior al daño mecánico observado. Babić et al. (2013) encontraron incrementos, decrementos y en general un comportamiento irregular de la fuerza a ruptura conforme se incrementó la humedad en semillas de maíz (Zea mays L.) de diferentes variedades.

Por otra parte, George et al. (2003) mencionan que el daño mecánico es de mayor intensidad en semillas de mayor tamaño, aunque la germinación también fue mayor; sin embargo, Moes y Vyn (1988) reportan que la semilla redonda y pequeña es la más susceptible al daño mecánico. Peterson et al. (1995) reportan que el estrato basal es el más susceptible al daño ocasionado por impacto en caída libre. Lundgren y Rosentrater (2007) encontraron que las semillas de especies de menor tamaño fueron más resistentes a compresión.

Bajo estos antecedentes, el objetivo de la presente investigación consistió en conocer la resistencia a compresión axial de la semilla del híbrido de maíz HS-2 de tres estratos de la mazorca a dos contenidos de humedad.

\section{MATERIALES Y MÉTODOS}

\section{Material genético y producción en campo}

Se utilizó el híbrido trilineal $\mathrm{HS}-2\left[\left(\mathrm{CL}_{12} \times \mathrm{CL}_{11}\right) \times \mathrm{CL}_{7}\right]$, desarrollado por el Dr. Aquiles Carballo Carballo, Profesor Investigador Titular del Programa de Semillas, Colegio de Postgraduados. El HS-2 es de grano blanco semidentado, adaptado a condiciones agroecológicas de los Valles Altos de México. La siembra de los progenitores se realizó en el ciclo primavera-verano de 2012 en el campo experimental del Colegio de Postgraduados, Montecillo, Estado de México, México. Se sembraron $5000 \mathrm{~m}^{2}$ en surcos a $80 \mathrm{~cm}$ y $50 \mathrm{~cm}$ entre plantas, se utilizó una relación de siembra 4:1 de hembra y macho. La siembra del macho se realizó el 27 de mayo, y la de la hembra $10 \mathrm{~d}$ después. Al momento de la siembra se fertilizó con la fórmula 120N-60P-00K. Veinticinco días después de antesis se realizaron muestreos a intervalos de $8 \mathrm{~d}$, en los que se cosecharon cinco mazorcas al azar para determinar la madurez de la semilla mediante la presencia de capa negra y línea de leche de acuerdo con la metodología descrita por Afuakwa y Crookston (1984).

\section{Dispositivo empleado en la prueba y variables evaluadas}

La compresión de las semillas se realizó con la máquina de ensayo universal Instron ${ }^{\circledR}$ modelo 4469, (Norwood, MA, USA) en el Departamento de Ingeniería Mecánica Agrícola (DIMA), Universidad Autónoma Chapingo. Se utilizaron superficies planas y pulidas (ASAE, 2005), y las semillas se colocaron en posición de descanso, con el embrión hacia arriba. La velocidad de la cruceta utilizada en todos los ensayos de compresión fue de $20 \mathrm{~mm} \mathrm{~min}^{-1}$. La célula de carga utilizada fue de $50 \mathrm{kN}$ (kilonewtons). El desplazamiento de la cruceta se fijó a $2 \mathrm{~mm}$, porque a esa distancia se presentaron todos los puntos característicos de la curva esfuerzo-deformación teóricos indicados por Mohsenin (1986). Al final se determinaron los promedios de la carga en $\mathrm{kN}$ y el desplazamiento en $\mathrm{mm}$ para cada punto característico.

\section{Factores de estudio}

\section{Contenido de humedad}

Se realizó la evaluación de la resistencia a compresión axial a dos contenidos de humedad (con base en peso húmedo): $23 \% \pm 0.5$ (después de madurez fisiológica), y $10 \%$ \pm 0.5 (después de secado artificial). Para el estudio de este factor se utilizaron únicamente semillas del estrato medio. El secado se realizó en un secador experimental del Programa de Semillas a $35^{\circ} \mathrm{C} \pm 1{ }^{\circ} \mathrm{C}$ con un flujo de aire de 43 $\mathrm{m}^{3} \mathrm{~s}^{-1}$. El contenido de humedad se determinó mediante el método de la estufa descrito por la ISTA (2005).

\section{Estrato en la mazorca}

Las muestras fueron extraídas de los estratos apical, medio y basal de la mazorca, para comparar la respuesta de la semilla a la compresión axial según su forma y tamaño. Los estratos apical y basal se conformaron por las semillas ubicadas en los primeros $4 \mathrm{~cm}$ a partir del extremo correspondiente, y el estrato medio se conformó por las hileras centrales restantes. Para el estudio de este factor, se utilizaron solamente semillas con $10 \%$ de humedad.

\section{Diseño del experimento y análisis estadístico}

Para estudiar el efecto producido por el contenido de humedad, se utilizó un diseño experimental completamente al azar con un factor de estudio (fuerza o desplazamiento), donde se compararon las magnitudes de la fuerza y desplazamiento en cada uno de los puntos característicos (LL, $Y$ y R) para ambos contenidos de humedad, para ello se realizó una prueba de medias de Tukey $(a=0.05)$. En este 
experimento se utilizaron tres muestras de 30 semillas del estrato medio por cada contenido de humedad.

Para estudiar el efecto producido por el estrato en la mazorca, se utilizó un diseño experimental completamente al azar con un factor de estudio (estrato en la mazorca). Se realizó una comparación de medias (Tukey, a $=0.05)$ para determinar si la fuerza o el desplazamiento soportados en cada punto característico de la curva carga-desplazamiento es superior en alguno de los tres estratos. En este experimento se utilizaron tres muestras de 30 semillas a $10 \%$ en contenido de humedad por cada estrato.

\section{RESULTADOS Y DISCUSIÓN}

\section{Fuerza y desplazamiento de semillas con $23 \%$ de humedad}

La curva de una de las semillas del estrato medio con $23 \%$ de humedad sometidas a compresión (Figura 1A), muestra similitud con la teoría descrita por Mohsenin (1986), aunque la ruptura del producto no coincidió con el punto en el que ésta ocurre ( $R^{\prime}$ ) según dicha teoría (donde la resistencia de la muestra a ser comprimida decae de manera drástica), sino que ocurrió a un desplazamiento menor (aproximadamente en el punto R; datos no mostrados); otras semillas del mismo estrato, en cambio, presentaron una curva carga-desplazamiento diferente a lo establecido a esa teoría (Figura 1B), en las que la ruptura también se presentó aproximadamente en el punto de inflexión posterior a biocedencia $(R)$, razón por la que dicho punto se consideró como el de ruptura.

En los estándares de la American Society of Agricultural Engineers (ASAE, 2005), la biocedencia se define como el punto en el que "un incremento en deformación no resulta en incremento en la fuerza", y no se menciona su relación con la ruptura de tejidos, por lo que la ruptura interna o externa puede o no ocurrir. Las muestras ensayadas presentaron similitud de apariencia y valores hasta los puntos $Y$ y $R$, posterior a los cuales, hubo una gran variación, como se observa en los gráficos de la Figura 1, aunque en éste y diversos estudios, como el de Murthy y Bhattacharya (1998), el comportamiento de la curva más allá de la ruptura no reviste de mucha importancia, especialmente por carecer de algún significado físico relevante. Con base en los resultados obtenidos y los estándares de la ASAE (2005), se considera a $Y$ simplemente como biocedencia, mientras que a $\mathrm{R}$ como ruptura, aunque en realidad corresponde a la fuerza y desplazamiento final de la "grieta rápida" (Ashby y Jones, 2009), mientras que el inicio de la fractura sucedió en Y. Los gráficos correspondientes a las semillas con $10 \%$ de hume- dad, fueron similares a los mostrados en la Figura 1.

El comportamiento de las curvas mostradas difiere en el segmento posterior a $\mathrm{R}$. Es probable que tanto biocedencia como ruptura hayan ocurrido en el mismo punto, en el marcado como Y; otra posibilidad es que en biocedencia solo iniciara el daño de la microestructura a nivel interno, y se propagara de manera continua hasta ocasionar la ruptura de las semillas, hecho que fue visible en R. Es también posible que las semillas presentaran un comportamiento diferente entre sí, como ocurrió en especímenes preparados de papa (Solanum tuberosum), donde los puntos de biocedencia y ruptura fueron monitoreados con el uso de emisiones acústicas (Zdunek y Konstankiewicz, 2004).

En cuanto a la diferencia en conformación de la curva carga-desplazamiento en los gráficos de la Figura 1, es probable que se deba a las propiedades mecánicas anisotrópicas de las semillas, además de las diferencias en dimensiones de las mismas, así como a las proporciones de endospermo duro, suave y embrión. Diferencias similares encontraron Zdunek y Konstankiewicz (2004) en curvas carga-desplazamiento correspondientes a especímenes preparados de papa, a pesar de las dimensiones uniformes.

Los análisis de varianza realizados para detectar diferencia significativa en las cargas y desplazamientos soportados en los tres puntos característicos de la curva cargadeformación (límite proporcional, biocedencia y ruptura), de las semillas sometidas a compresión con 23 y $10 \%$ de humedad, indican que no todos los puntos característicos de la curva carga-deformación se presentaron en la misma carga ( $P \leq 0.05$ y $P \leq 0.01$, respectivamente), ni a desplazamientos iguales $(P \leq 0.05$ y $P \leq 0.01)$, de manera significativa (Cuadro 1).

La prueba de medias de Tukey realizada para semillas con $23 \%$ de humedad mostró que ruptura y biocedencia no son significativamente diferentes ni en carga ni en desplazamiento; mientras que límite proporcional y ruptura son significativamente diferentes en desplazamiento, y existe diferencia significativa entre los puntos de límite proporcional y biocedencia en la carga soportada. La prueba de comparación de medias de Tukey realizada para semillas con humedad de $10 \%$, mostró que los desplazamientos en los tres puntos característicos de la curva carga-desplazamiento fueron significativamente diferentes entre sí, mientras que la carga soportada en biocedencia fue significativamente superior al límite proporcional y a la ruptura (Figura 2) sin que la carga entre límite proporcional y ruptura fuera significativamente diferente. 

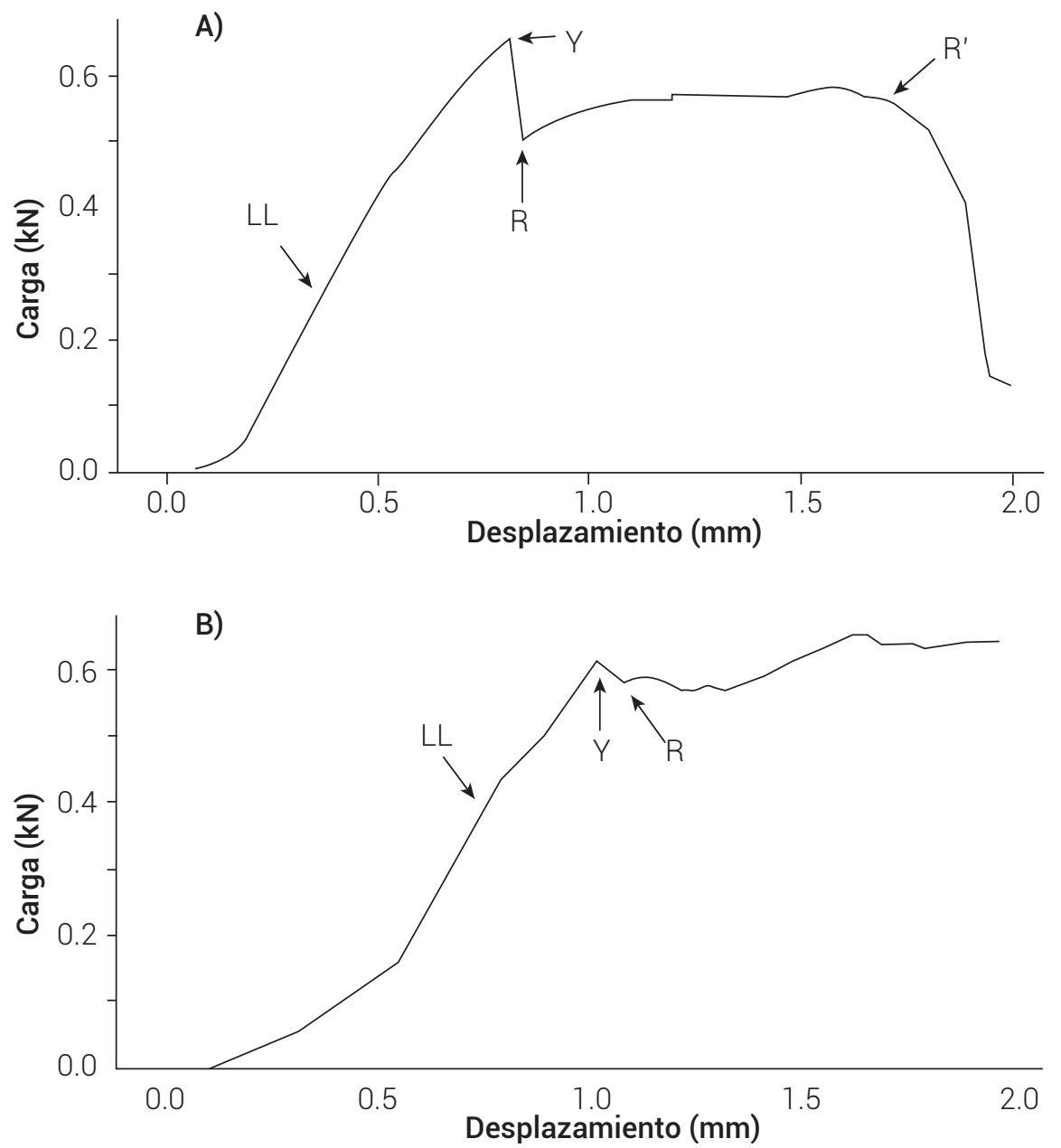

Figura 1. Curvas carga-desplazamiento de dos semillas con $23 \%$ de humedad del estrato medio, la ruptura ocurrió aproximadamente en $\mathrm{R}$; Y y LL corresponden a biocedencia y límite proporcional.

Los promedios de desplazamiento $(\mathrm{mm})$ y carga $(\mathrm{kN})$ en cada punto característico de la curva desplazamientocarga, en semillas del estrato medio con humedad de 10 y $23 \%$ (base húmeda), fueron de: (0.215C, 0.301b) y (0.827B, 0.429b) en límite proporcional LL, $(0.303 \mathrm{~B}, 0.434 \mathrm{a})$ y (1.165AB, 0.598a) en biocedencia $Y$ y $(0.341 \mathrm{~A}, 0.344 \mathrm{~b})$ y (1.215A, 0.568ab) en ruptura $R$; donde las medias con misma letra para cada punto: $L L, Y$ y $R$, no difieren estadísticamente (Tukey, 0.05; DMSH 0.017 y 0.339 para valores de desplazamiento y 0.044 y 0.151 para valores de carga a 10 y $23 \%$ de contenido de humedad, letras mayúsculas se utilizaron para comparar las medias de desplazamiento y letras minúsculas para comparar las medias de carga. Es evidente que las semillas con humedad de $23 \%$ fueron superiores en los valores promedio de carga y desplazamiento en cada punto crítico (Tukey, 0.05).

Las semillas del estrato medio con $23 \%$ de humedad fueron más elásticas y resistieron más fuerza a ruptura que las semillas con humedad menor de $10 \%$ (Figura
2), lo que concuerda con lo mencionado por Bosoi et al. (1991), aunque es probable que la relación entre humedad y resistencia a ruptura no tenga un comportamiento lineal (Mohsenin, 1986). Además, los daños de magulladuras a mayor contenido de humedad pueden repercutir en la posterior disminución de la calidad fisiológica de las semillas (Paulsen, 1978). De acuerdo con Bosoi et al. (1991), el enlace entre las semillas y la mazorca se incrementa con el contenido de humedad de manera proporcional, por lo que el daño mecánico ocasionado durante la trilla por exceso de secado, se atribuye a la menor resistencia de la semilla, mientras que el daño mecánico por exceso de humedad, estará atribuido a la mayor fuerza de enlace entre la semilla y la mazorca, además de la acción de fuerzas cortantes.

\section{Comparación de la resistencia a compresión axial de semillas de los estratos con $10 \%$ de humedad}

El desplazamiento para alcanzar el límite proporcional (LL) no difiere significativamente entre los estratos (Figura 3); 
mientras que para alcanzar biocedencia $(\mathrm{Y})$ y ruptura $(\mathrm{R})$ el estrato medio fue superior al estrato apical únicamente.

La semilla del estrato medio ofreció mayor resistencia en el punto de biocedencia ( $\mathrm{Y})$, mientras que para alcanzar LL y R solo fue superior al estrato basal (Figura 3). Debido a que las semillas del estrato medio soportaron la mayor carga a biocedencia, se puede decir que es el estrato más resistente a compresión, lo que lo hace menos susceptible a daño mecánico durante el proceso de desgrane, aunado a ello, la fuerza de enlace con la mazorca es inferior a la de los estratos apical y basal (Bosoi et al., 1991). Este resultado podría contrastar con los obtenidos por George et al. (2003) quienes encontraron mayor daño en semillas grandes (clasificadas por ancho), en vista de que éstas se concentran en la porción central de la mazorca (Batistella et al., 2002); sin embargo, los resultados obtenidos por George et al. (2003) pueden atribuirse a que las semillas de la porción central maduran antes que las del ápice y de la base (Bosoi et al., 1991) y pueden perder humedad en menos tiempo,

Cuadro 1. Análisis de varianza para la carga y el desplazamiento, en los puntos característicos de la curva cargadesplazamiento de semillas comprimidas con 23 y $10 \%$ de humedad. Chapingo, México. 2013.

\begin{tabular}{|c|c|c|c|c|c|c|}
\hline FV & $\mathrm{GL}$ & SC & $\mathrm{CM}$ & Fc & Valor $p$ & Significancia \\
\hline \multicolumn{7}{|c|}{ Semillas con $23 \%$ de humedad } \\
\hline Desplazamiento & 2 & 0.2677 & 0.1338 & 7.33 & 0.0245 & * \\
\hline Error & 6 & 0.1096 & 0.0183 & & & \\
\hline Total & 8 & 0.3773 & & & & \\
\hline Carga & 2 & 0.0482 & 0.0241 & 6.59 & 0.0306 & * \\
\hline Error & 6 & 0.0219 & 0.0037 & & & \\
\hline Total & 8 & 0.0701 & & & & \\
\hline \multicolumn{7}{|c|}{ Semillas con $10 \%$ de humedad } \\
\hline Desplazamiento & 2 & 0.0251 & 0.012532 & 276.44 & $<0.0001$ & ** \\
\hline Error & 6 & 0.0003 & 0.00004 & & & \\
\hline Total & 8 & 0.0253 & & & & \\
\hline Carga & 2 & 0.0274 & 0.0137 & 43.93 & 0.0003 & ** \\
\hline Error & 6 & 0.0019 & 0.0003 & & & \\
\hline Total & 8 & 0.0292 & & & & \\
\hline
\end{tabular}

**Significativo (P $\leq 0.01)$; *Significativo $(\mathrm{P} \leq 0.05)$; FV: factor de variación; GL: grados de libertad; SC: suma de cuadrados; CM: cuadrados medios del error; Fc: valor calculado de F.

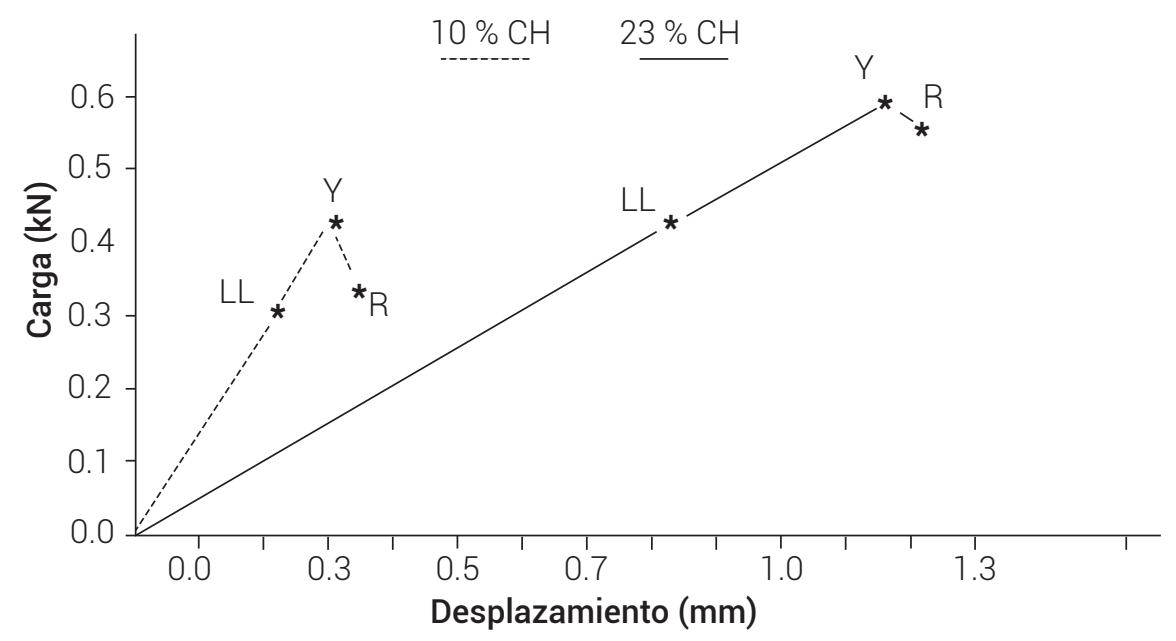

Figura 2. Promedios de desplazamiento $(\mathrm{mm})$ y carga $(\mathrm{kN})$ en cada punto característico de la curva desplazamientocarga, unidos por líneas hipotéticas, en semillas del estrato medio con humedad de 10 y $23 \%$ (base húmeda). 


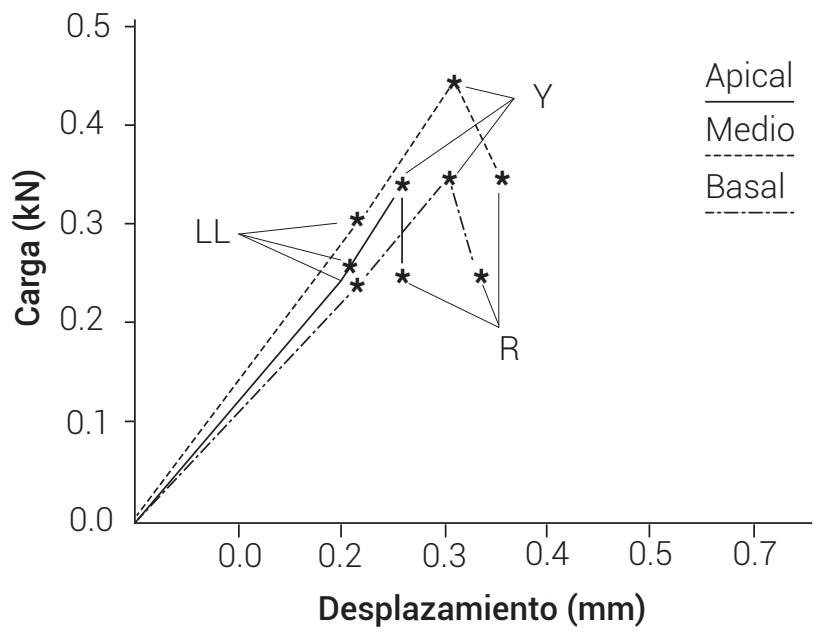

Figura 3. Promedios de desplazamiento y carga en cada punto característico de la curva desplazamiento-carga: límite lineal LL, biocedencia Y y ruptura R. Las líneas que unen los puntos son hipotéticas. Valores correspondientes a semillas con humedad de $10 \%$, de los estratos apical, medio y basal de la mazorca.

para hacerse más susceptibles a las cargas propiciadas por el desgrane; el procedimiento utilizado en el presente trabajo elimina la variación de la humedad entre estratos debida a la madurez diferencial de éstos.

De acuerdo con los resultados de este estudio, podría ser conveniente realizar el desgrane en dos etapas, en donde la primera tenga el objetivo de desprender las semillas de la parte central, dada la susceptibilidad de las semillas y el débil enlace con la mazorca por el bajo contenido de humedad; y la segunda, las de las porciones apical y basal (pues la resistencia de la semilla es mayor al igual que el enlace con la mazorca, debido a un mayor contenido de humedad; mediante los regímenes de trabajo adecuados en cada etapa para garantizar el menor daño mecánico, y por lo tanto una mejor calidad de la semilla.

Los valores promedio en pares ordenados del desplazamiento $(\mathrm{mm})$ y carga $(\mathrm{kN})$ para semillas del estrato apical, medio y basal fueron: (0.206A, 0.258ab), (0.215A, 0.301a) y $(0.213 \mathrm{~A}, 0.238 \mathrm{~b})$ en el límite proporcional LL, $(0.258 \mathrm{~B}$, $0.345 b),(0.303 \mathrm{~A}, 0.434 \mathrm{a})$ y $(0.296 \mathrm{~A}, 0.341 \mathrm{~b})$ en biocedencia $Y$, y $(0.258 \mathrm{~B}, 0.248 \mathrm{ab}),(0.341 \mathrm{~A}, 0.344 \mathrm{a})$ y $(0.325 \mathrm{AB}$, $0.246 \mathrm{~b}$ ) en ruptura $\mathrm{R}$ (Figura 3). Se utilizaron letras mayúsculas para comparar las medias de desplazamiento y letras minúsculas para comparar las medias de carga, donde medias con misma letra para cada punto: $L L, Y$ y $R$, no difieren estadísticamente (Tukey, 0.05; DSH 0.023, 0.037 y 0.047 para valores de desplazamiento y $0.05,0.056$ y 0.097 para valores de carga).

\section{CONCLUSIONES}

Las semillas de maíz del híbrido HS-2 son más resistentes a las cargas estáticas y más elásticas a mayor contenido de humedad. La carga en biocedencia fue superior a la carga en límite proporcional para ambos contenidos de humedad, y a la carga en ruptura, para semillas con humedad de $10 \%$. En semillas con $23 \%$ de humedad, no hubo diferencia significativa entre el desplazamiento a biocedencia y el de límite proporcional, ni entre el de biocedencia y ruptura, pero sí entre el de ruptura y límite proporcional. Hubo diferencia significativa en el desplazamiento a límite proporcional, biocedencia y ruptura para semillas con 10 $\%$ de humedad. Las semillas del estrato medio fueron más resistentes a las cargas que las semillas que los estratos apical y basal, según la carga soportada en biocedencia; también resultaron más resistentes que las semillas del estrato basal en las cargas a límite proporcional y a ruptura, pero no fueron más resistentes que las del estrato apical a las cargas en límite proporcional y ruptura.

\section{BIBLIOGRAFÍA}

Afuakwa J. J. and R. K. Crookston (1984) Using the kernel milk line to visually monitor grain maturity in maize. Crop Science 24:687-691.

ASAE, American Society of Agricultural Engineers (2005) Standard S368.4 DEC00: Compression Test of Food Materials of Convex Shape. ASAE Standards 2005. 52nd ed. Standards Engineering Practices Data. St. Joseph, MI, USA. pp:601-608.

Ashby M. F. y D. R. H. Jones (2009) Materiales para Ingeniería 2. Introducción a la Microestructura, el Procesamiento y el Diseño. Editorial Reverté. Barcelona, España. 510 p.

Babić L. J., M. Radojčin, I. Pavkov, M. Babić, J. Turan, M. Zoranović and S. Stanišić (2013) Physical properties and compression loading behaviour of corn seed. International Agrophysics 27:119-126.

Basu R. N. (1995) Seed viability. In: Seed Quality: Basic Mechanisms 
and Agricultural Implications. A. S. Basra (ed.). Food Products Press. New York, USA. pp:1-44.

Batistella F., F. V. Moro and N. M. De Carvalho (2002) Relationship between physical, morphological, and physiological characteristics of seeds developed at different positions of the ear of two maize (Zea mays L.) hybrids. Seed Science and Technology 30:97-106.

Bosoi E. S., O. V. Verniaev, I. I. Smirnov and G. E. Sultan-Shakh (1991) Theory, Construction and Calculations of Agricultural Machines. Vol. 2. Translated from Russian by A. Jaganmohan. A. A. Balkema/ Rotterdam Ed., Rotterdam, The Netherlands. 1947 p.

Desai B. B., P. M. Kotecha and D. K. Salunkhe (1997) Seeds Handbook: Biology, Production, Processing, and Storage. Marcel Dekker. New York, USA. $627 \mathrm{p}$

García-Lara S., D. J. Bergvinson, A. J. Burt, A. I. Ramputh, D. M. Díaz-Pontones and J. T. Arnason (2004) The role of pericarp cell wall components in maize weevil resistance. Crop Science 44:1546-1552.

George D. L., M. L. Gupta, D. Tay and I. G. M. A. Parwata (2003) Influence of planting date, method of handling and seed size on supersweet sweet corn seed quality. Seed Science and Technology 31:351-366.

ISTA, International Seed Testing Association (2005) International Rules for Seed Testing. Edition 2005. Bassersdorf, Switzerland. pp:85-90

King D. L. and A. W. Riddolls (1960) Damage to wheat seed and pea seed in threshing. Journal of Agicultural Engineering Research 5:387-398.

Lundgren J. G. and K. A. Rosentrater (2007) The strength of seeds and their destruction by granivorous insects. Arthropod-Plant Interactions 1:93-99.

Mesquita C. M. and M. A. Hanna (1993) Soybean threshing mechanics: II Impact. Transactions of the American Society of Agricultural Engineers 36:281-284.
Moes J. and T. J. Vyn (1988) Management effects on kernel breakage susceptibility of early maturing corn hybrids. Agronomy Journal 80:699-704.

Mohsenin N. N. (1986) Physical Properties of Plant and Animal Materials. Structure, Physical Characteristics and Mechanical Properties. 2nd ed. Gordon and Breach Science Publishers. New York. $891 \mathrm{p}$.

Multon J. L., H. Bizot, J. L. Doublier, J. Lefebvre and D. C. Abbot (1981) Effect of water activity and sorption hysteresis on rheological behavior of wheat kernels. In: Water Activity: Influences on Food Quality. L. B. Rockland and G. F. Stewart (eds.). Academic Press. New York, USA. pp:179-198.

Murthy C. T. and S. Bhattacharya (1998) Moisture dependant physical and uniaxial compression properties of black pepper. Journal of Food Engineering 37:193-205

Narayan C. V. and W. K. Bilanski (1966) Behavior under high pressure of wheat grains in bulk. Transactions of the American Society of Agricultural Engineers 13:298-302.

Paulsen M. R. (1978) Fracture resistance of soybeans to compressive loading. Transactions of the American Society of Agricultural Engineers 21:1210-1216

Peterson J. M., J. A. Perdomo and J. S. Burris (1995) Influence of kernel position, mechanical damage and controlled deterioration on estimates of hybrid maize seed quality. Seed Science and Technology 23:647-657.

Poehlman J. M. y D. A. Sleper (2003) Mejoramiento Genético de las Cosechas. Editorial Limusa. México, D. F. 506 p.

Zdunek A. and K. Konstankiewicz (2004) Acoustic emission in investigation of plant tissue micro-cracking. Transactions of the American Society of Agricultural Engineers 47:1171-1177. 